\section{Influenza Vaccination Rates, Feedback, and the Hawthorne Effect}

TO THE EDITOR-For the past 2 decades, the Centers for Disease Control and Prevention has recommended annual influenza vaccination for all healthcare workers. Yet nationally, only $40 \%$ of healthcare workers are vaccinated each year. ${ }^{1}$ Hospitals and clinics have used a variety of approaches to increase vaccination rates. The most effective measures have included providing vaccination for free and at times and sites that are convenient for the staff. Institutions that do not promote the use of influenza vaccine report influenza vaccination rates of only $5 \%-19 \%$, compared with rates of $26 \%$ $54 \%$ for institutions that have active vaccination programs. ${ }^{2}$ Despite the success of some influenza campaigns, the intensity of the programs needs to be maintained from year to year, and progress can be undermined if key personnel are no longer available. ${ }^{3}$ Furthermore, efforts to increase vaccination rates, including making the vaccine readily available, have not been universally successful. ${ }^{4}$ Given the persistently low influenza vaccination rates and the significant cost of some vaccination programs, low-cost methods for increasing compliance are needed. We propose that providing information on group vaccination rates to supervisors may be an efficient and cost-effective means for hospitals to increase vaccination rates among healthcare workers.

To encourage influenza vaccination, the University of Iowa Hospital and Clinics offers free influenza vaccination for 4 weeks during an influenza vaccination campaign each fall. In 2003, we assessed vaccination rates for each clinical department and treatment unit at the University of Iowa Hospital and Clinics after the vaccine campaign had been completed. Because vaccination rates were low, the free influenza vaccination campaign was extended for an additional 4 weeks; all hospital employees were informed of this extension through numerous e-mail messages and posters. The residency program directors or chief residents of the internal medicine, family practice, obstetrics and gynecology, general surgery, neurology, and orthopedics departments were informed of the influenza vaccination rates for their residents (the intervention group; $n=195$ ). In addition, we offered the vaccine at the departmental conferences for some of the residency programs (medicine, family practice, obstetrics and gynecology, and neurology). The control group (residents in pediatrics, anesthesiology, dermatology, neurosurgery, otolaryngology, psychiatry, radiation oncology, and urology; $\mathrm{n}=176$ ) was exposed only to e-mail messages and posters promoting the influenza vaccination campaign; the residency program directors or chief residents of these departments were not informed of the vaccination rates for their residents, and we did not offer the vaccine at their departmental conferences. The Institutional Review Board of the University of Iowa Hospital and Clinics approved the analysis and reporting of the present results. We used a 1-tailed Fisher's exact test to determine whether the increase in vaccination rates differed between the intervention group and the control group. A 1-tailed test was used because vaccination rates could only increase among this population-the healthcare workers could not become "unvaccinated." All statistical analyses were performed using Stata (version 8; StataCorp).

We found that the vaccination rate increased $38 \%$ in the intervention group, compared with only $13 \%$ in the control group ( $P=.04$ ). Thirty-one additional residents were vaccinated in the intervention group (the total increased from 82 to 113 ), compared with just 13 additional residents in the control group (the total increased from 95 to 108). Offering vaccine at resident conferences did not appear to significantly increase the effectiveness of this intervention among resident physicians $(P=1.00)$.

A simple intervention, providing vaccination rates to residency program directors or chief residents, increased influenza vaccination rates in the intervention group. In an era of decreasing resources, with growing populations at risk for complications of influenza, healthcare facilities need new interventions to increase vaccination rates. Evaluating vaccination rates halfway through a vaccination campaign and reporting the results to supervisors-or perhaps to all employees-could be a useful method for increasing the use of the vaccine.

Contrary to expectations, offering vaccine at departmental conferences did not increase vaccination rates. However, a much larger sample size might have revealed a statistically significant difference. Because we did not have information on individual rates for conference attendees versus nonattendees, we cannot rule out the possibility that residents who regularly attend departmental conferences may be more likely to attend the scheduled vaccination clinics. Providing easy access to the vaccine has usually been associated with increased vaccination rates, although this has not always been the case. ${ }^{4}$

Our study was designed as a process-improvement project to increase vaccination rates quickly with limited resources. Most of the limitations of this study result from its design. One is that we did not select the groups of residents randomly (future studies should do so). We intentionally included larger residency programs with lower vaccination rates in the intervention group. However, no one department was responsible for the positive results of this study. Among the intervention group, the percent change in vaccination rates among groups of residents ranged from $14 \%$ to $38 \%$, with an average of $27 \%$.

More primary care residents were included in the inter- 
vention group, and it is possible that this group was inherently more likely to accept the influenza vaccine. However, we found low vaccination rates among internal medicine $(43 \%)$ and family practice $(46 \%)$ residents before the vaccination program was extended. Interestingly, dermatology residents had the highest vaccination rate (88\%) among the residency training programs, and vaccination rates in housekeeping (55\%) and in maintenance and engineering (65\%) exceeded those for most groups of nurses and residents at the University of Iowa Hospital and Clinics. Unfortunately, no information on what different supervisors did with the vaccination data is available. Finally, our project specifically targeted residents, and, in the future, other hospital staff should be included.

Feeding back information to physicians has been shown to positively affect physician behavior..$^{5-7}$ However, we did not inform the residents directly of the vaccination rates; instead, we informed their supervisors. Because we did not feed information back to the residents directly, part of our treatment effect may have been the result of a Hawthorne effect. The Hawthorne effect refers to the observation that individual and group behaviors are often altered when individuals or groups know that they are being studied. Investigators have previously suggested that the Hawthorne effect be used to change the behavior of healthcare workers in the desired direction. ${ }^{8-10}$ Informing healthcare workers that their vaccination status is being observed may help to increase vaccination rates. In the future, we hope to assess the effect of communicating this information both at the group level and at the individual level.

Our targeted intervention increased influenza vaccination rates. The critical components of this intervention included informing departments that their vaccination rates were being monitored and providing supervisors with the vaccination rates of their residents. Healthcare epidemiologists could use this inexpensive approach to increase influenza vaccination rates among healthcare workers.

Philip M. Polgreen, MD; Jean Pottinger, MA; Linnea A. Polgreen, PhD; Daniel J. Diekema, MD; Loreen A. Herwaldt, MD

Drs. P. M. Polgreen, Diekema, and Herwaldt are from the Division of Infectious Diseases, Department of Internal Medicine, University of Iowa Carver College of Medicine. Ms. Pottinger is from the Program of Hospital Epidemiology, University of Iowa Hospitals and Clinics, and Dr. L. A. Polgreen is from the Department of Economics, University of Iowa Tippie College of Business. Drs. Diekema and Herwalt are also from the Program of Hospital Epidemiology, University of lowa Hospitals and Clinics, and Dr. Diekema is from the Division of Medical Microbiology, Department of Pathology, University of Iowa Carver College of Medicine, and the Iowa City Veterans Affairs Medical Center as well.

Infect Control Hosp Epidemiol 2006; 27:98-99

(C) 2006 by The Society for Healthcare Epidemiology of America. All rights reserved. 0195-9417/2006/2701-0023\$15.00.

\section{REFERENCES}

1. Harper SA, Fukuda K, Uyeki TM, Cox NJ, Bridges CB. Prevention and control of influenza: recommendations of the Advisory Committee on Immunization Practices (ACIP). Centers for Disease Control and Prevention. MMWR Recomm Rep 2004; 53:1-40.

2. Pottinger JM, Herwaldt LA. Improving health care worker compliance with influenza immunization. In: Poland GA, Schaffner W, Pugliese G, eds. Immunizing Health Care Workers: A Practical Approach. Thorofare, NI: Slack; 2000.

3. Fedson DS. Influenza vaccination of medical residents at the University of Virginia: 1986-1994. Infect Control Hosp Epidemiol 1996; 17:431-433.

4. Pachucki CT, Pappas SWA, Fuller GF, Krause SL, Lentino JR, Schaaff DM. Influenza A among hospital personnel and patients: implications for recognition, prevention, and control. Arch Intern Med 1989; 149:77-80.

5. Cruse PJ, Foord R. The epidemiology of wound infection: a 10-year prospective study of 62,939 wounds. Surg Clin North Am 1980; 60:27-40.

6. Haley RW, Culver DH, White JW, et al. The efficacy of infection surveillance and control programs in preventing nosocomial infections in US hospitals. Am J Epidemiol 1985; 121:182-205.

7. MacDonald A, Dinah F, MacKenzie D, Wilson A. Performance feedback of hand hygiene, using alcohol gel as the skin decontaminant, reduces the number of inpatients newly affected by MRSA and antibiotic costs. $J$ Hosp Infect 2004; 56:56-63.

8. Feil PH, Grauer IS, Gadbury-Amyot CC, Kula K, McCunniff MD. Intentional use of the Hawthorne effect to improve oral hygiene compliance in orthodontic patients. J Dent Educ 2002;66: 1129-1135.

9. Mangione-Smith R, Elliott MN, McDonald L, McGlynn EA. An observational study of antibiotic prescribing behavior and the Hawthorne effect. Health Serv Res 2002; 37:1603-1618.

10. Lied TR, Kazandjian VA. A Hawthorne strategy: implications for performance measurement and improvement. Clin Perform Qual Health Care 1998; 6:201-204.

\section{Prevalence of Needlestick Injuries Among Medical Students at a University in Iran}

TO THE EDITOR-The incidence of human immunodeficiency virus (HIV) infection in Iran is increasing at an alarming rate, especially among intravenous drug abusers. In different areas of Iran, the incidence of hepatitis $B$ virus (HBV) infection ranges from $1.75 \%$ to $5 \%$, and the incidence of hepatitis $\mathrm{C}$ virus (HCV) infection ranges from $0.2 \%$ to $1.5 \%{ }^{1,2}$ Among the most serious occupational hazards for medical students are injuries from sharp instruments and devices (sharps) and needlestick injuries (NSIs) and the associated risk of exposure to bloodborne viruses, including $\mathrm{HBV}, \mathrm{HCV}$, and HIV.,4

The rate of acquisition of a bloodborne virus depends on the prevalence of the virus among the patient population and the susceptibility of the health care worker. ${ }^{5}$ Recent estimates of rates of acquisition among susceptible health care workers were $6 \%$ to $30 \%$ for $\mathrm{HBV}, 5 \%$ to $10 \%$ for $\mathrm{HCV}$, and $0.3 \%$ for HIV. ${ }^{6}$ Administration of preexposure vaccination or prophylaxis is more than $90 \%$ effective in the prevention of $\mathrm{HBV}$ infection, less effective in the prevention of HIV infection, and not effective in the prevention of HCV infection. 